\title{
Wirksamkeit einer kombinierten Therapie der atopischen Dermatitis mit Triclosan und Flumetasonpivalat (DuoGalen ${ }^{\circledR}$ Creme)
}

\author{
Efficacy of a Combined Treatment of Atopic Dermatitis with Triclosan \\ and Flumethasone Pivalate (DuoGalen ${ }^{\circledR}$ Cream)
}

Autoren

Institute
I. Zschocke ${ }^{1}$, A. Lotzin ${ }^{1}$, M. Sebastian² ${ }^{2}$ T. Dirschka ${ }^{3}$, A. Zielke ${ }^{1}$, K. Reich ${ }^{1,4}$

SClderm GmbH, Hamburg

Facharztpraxis für Dermatologie, Mahlow

Facharztpraxis für Dermatologie, Wuppertal

Dermatologikum Hamburg

\section{Bibliografie}

Dol http://dx.doi.org/

10.1055/s-0029-1214754

Online-Publikation: 30. 6. 2009

Akt Dermatol 2009; 35:

496-501 @ Georg Thieme

Verlag KG Stuttgart · New York ISSN 0340-2541

\section{Korrespondenzadresse}

Dr. Ina Zschocke

SClderm GmbH

Esplanade 6

20354 Hamburg

ina.zschocke@sciderm.com

\section{Zusammenfassung}

Ziel der Studie: Ziel der Anwendungsbeobachtung war die Abschätzung der Wirksamkeit und des patientenseitigen Nutzens der Behandlung der atopischen Dermatitis mit einer fixen Kombination des Antiseptikums Triclosan und Flumetasonpivalat.

Patienten und Methodik: 31 Patienten mit leichter bis mittelschwerer atopischer Dermatitis wurden in eine Anwendungsbeobachtung eingeschlossen, bei der Triclosan/Flumetasonpivalat über 2 Wochen zweimal täglich appliziert wurde. Es erfolgte eine Bestimmung des Schweregrads der klinischen Symptome und der mikrobiellen Besiedlung in einem Prüfareal. Die Patienten bewerteten Wirksamkeit, Verträglichkeit und subjektiven Nutzen der Therapie sowie die krankheitsbezogene Lebensqualität.

\section{Einleitung}

Die atopische Dermatitis ist eine chronische oder chronisch-rezidivierende entzündliche Hauterkrankung, die mit starkem Juckreiz einhergeht. Sie kann in jedem Lebensalter auftreten, besonders häufig ist sie im Kindes- und Jugendalter [1]. Die Prävalenz der atopischen Dermatitis bis zum Schulanfang beträgt 10-15\% [2]. Der Verlauf der Erkrankung ist wechselhaft mit Krankheitsschüben unterschiedlicher Dauer und Schwere [1]. Insbesondere bei schwereren Formen der Erkrankung kann die atopische Dermatitis zu einer deutlichen Minderung der Lebensqualität führen $[3,4]$. Die Diagnose erfolgt klinisch anhand der typischen Morphologie der Hautveränderungen, des chronisch oder chronisch-rezidivierenden Verlaufs, der Eigen- und Familienanamnese und des begleitenden Juckreizes [1]. Ätiologisch spielen sowohl eine genetische Prädisposition zu atopischen Erkrankungen (allergisches Asthma bronchiale, Rhinokonjunktivitis) sowie verschie-
Ergebnisse: Der klinische Symptomscore $(0=$ keine, 1 = leichte, 2 = mäßige, 3 = starke Symptome) verringerte sich signifikant im Verlauf der Triclosan/Flumetasonpivalat-Behandlung (vor Therapie: 1,14 $\pm 0,39$; Therapieende: $0,43 \pm 0,29$; $p<0,001)$. Parallel zeigte sich eine Reduktion der mit Staphylococcus aureus infizierten Prüfareale (Abnahme von 55\% auf 28\%; $p<0,01$ ). Die Wirksamkeit der Therapie wurde von den Patienten im Mittel als „gut“, die Verträglichkeit als „gut“ bis „sehr gut“ bewertet. Die Lebensqualität besserte sich deutlich (DLQI; vor Therapie: $8,13 \pm 4,78$; Therapieende: $3,21 \pm 3,59$ ).

Schlussfolgerungen: Die Ergebnisse deuten auf eine gute klinische und mikrobiologische Wirksamkeit von Triclosan/Flumetasonpivalat in der Therapie der atopischen Dermatitis bei guter Verträglichkeit und Patientenzufriedenheit hin. dene Umweltfaktoren eine Rolle [5]. Sebostase, $\mathrm{pH}$-Anomalien und eine Barrierefunktionsstörung der Haut sowie Störungen der zellulären und humoralen Immunantwort tragen zu einer Superinfektion mit Staphylococcus (Staph.) aureus bei, die bei 80 - 100\% der Patienten mit atopischer Dermatitis nachzuweisen ist [6-11] und als Triggerfaktor angesehen wird [11]. Das Ausmaß der Superinfektion korreliert dabei mit dem Schweregrad der atopischen Dermatitis [11-13].

Zur Lokaltherapie der atopischen Dermatitis werden antiseptische, antibiotische, antimykotische, antipruriginöse und antiinflammatorische Substanzen eingesetzt [14]. Die topischen Steroide zählen mit ihrer guten anti-entzündlichen Wirkung zu den Therapieoptionen der ersten Wahl [14]. Monotherapien mit topischen Steroiden sind aber häufig nicht ausreichend wirksam [1]. Bei einer Besiedlung mit Staph. aureus führt eine zusätzliche antimikrobielle Therapie mit Antiseptika oder Antibiotika zu einer signifikanten Besserung der atopischen Dermatitis $[6,10,11]$. Die ef- 
fektive antimikrobielle Behandlung scheint zudem die pathologische Immunantwort der betroffenen Haut zu reduzieren [11]. Zur Vermeidung von Resistenzentwicklungen in der antimikrobiellen Therapie ist der Einsatz von Antiseptika der Anwendung von Antibiotika vorzuziehen [14,15]. Triclosan spielt als Breitspektrumantiseptikum bei der Behandlung der atopischen Dermatitis aufgrund seiner guten Wirksamkeit gegen Staph. aureus eine wichtige Rolle. Die Substanz ist gut verträglich, nicht-karzinogen, nicht-mutagenen und nicht-teratogen [16]. Resistenzen wurden bisher nur vereinzelt beschrieben [17].

Kombinationspräparate mit Triclosan und Flumetasonpivalat werden vor allem bei entzündlichen und mykotisch oder bakteriell infizierten entzündlichen Dermatosen eingesetzt. Verschiedene Veröffentlichungen berichten über gute Erfahrungen mit Kombinationspräparaten aus Triclosan und Flumetasonpivalat bei der Behandlung akuter und subakuter, bakteriell und/oder mykotisch infizierter Ekzeme und akuter oberflächlicher Dermatomykosen bei Patienten verschiedener Altersstufen [18-22]. DuoGalen ${ }^{\circledR}$-Creme enthält Triclosan in 3\%iger Konzentration sowie Flumetasonpivalat in 0,02\%iger Konzentration in einer $\mathrm{O} / \mathrm{W}$ Emulsion [16]. Bei zweimal täglicher Anwendung von Triclosan/ Flumetasonpivalat kann eine Abheilung oder deutliche Besserung des klinischen Befundes bereits nach 2 Wochen erwartet werden $[19,20]$.

Primäres Ziel dieser gemäß den Empfehlungen der Deutschen Gesellschaft für Medizinische Informatik, Biometrie und Epidemiologie (GMDS) [23] durchgeführten Anwendungsbeobachtung war es, die klinische Wirksamkeit sowie die antimikrobielle Aktivität des Kombinationspräparates DuoGalen ${ }^{\circledR}$-Creme unter Praxisbedingungen zu ermitteln. Als weitere Zielkriterien wurden die patientenseitige Einschätzung der Wirksamkeit und Verträglichkeit, der patientendefinierte Therapienutzen sowie die krankheitsbezogene Lebensqualität untersucht.

\section{Patienten und Methoden}

$\nabla$

Bei der vorliegenden multizentrischen, nicht kontrollierten, unverblindeten Kohortenstudie handelt es sich gemäß §67 Abs. 6 AMG um eine Anwendungsbeobachtung. Die Anwendungsbeobachtung wurde in zwei dermatologischen Praxen durchgeführt, die insgesamt 31 Patienten mit atopischer Dermatitis einschlossen. Die Therapie der betroffenen Hautstellen mit Triclosan/Flumetasonpivalat erfolgte zweimal täglich über einen Zeitraum von zwei Wochen. Die Erhebung der Prüfgrößen erfolgte vor der Behandlung, nach 7 Tagen Behandlungsdauer sowie zum Behandlungsende nach 14 Tagen.

Eingeschlossen wurden Patienten ab sechs Jahren, die an einem leichten bis mittelschweren atopischen Ekzem mit vorwiegend umschriebenen typischen Hautveränderungen an den Prädilektionsstellen (z. B. Hals, Armbeugen, Kniebeugen) litten. Die Unterscheidung in leichte, mittelschwere und schwere atopische Dermatitis wurde anhand des SCORAD (SCORing Atopic Dermatitis) [23] getroffen, wobei ein SCORAD von 1 bis 25 Punkten als leichte und ein SCORAD von 26 bis 50 Punkten als mittelschwere atopische Dermatitis gewertet wurde.

Ausgeschlossen wurden Patienten mit überwiegend xerotischen oder xerotisch-hyperkeratotischen Hautveränderungen, gleichzeitiger oder in den letzten 4 Wochen angewendeter systemischer anti-entzündlicher Medikation bzw. gleichzeitiger Lichttherapie, Überempfindlichkeit gegen einen der getesteten Wirkstoffe, Verdacht auf oder Diagnose eines Eczema herpeticatum sowie
Schwangerschaft oder Stillzeit. Eine begleitende Pflegetherapie einschließlich Urea bis zu einer Konzentration von 5\% war erlaubt. Bei den eingeschlossenen Patienten wurde ein für die Erkrankungsschwere repräsentatives Prüfareal festgelegt. Die Ausprägung der sechs Symptome Erythem, Ödem-/Papelbildung, Nässen-/Krustenbildung, Exkoriation, Lichenifikation und Trockenheit im Prüfareal wurde jeweils auf einer Skala von 0 bis 3 ( 0 = keine, 1 = leichte, 2 = mäßige, 3 = starke Symptome) quantifiziert. Die mittlere Schwere der sechs klinischen Symptome wurde als Hauptzielkriterium der Anwendungsbeobachtung definiert. Darüber hinaus wurde vor Behandlungsbeginn, nach 7 Tagen sowie zum Behandlungsende (Tag 14) ein mikrobiologischer Abstrich aus dem Prüfareal entnommen und mit mikrobiologischen Standardverfahren auf das Vorhandensein von grampositiven und gramnegativen Bakterien getestet. Die Veränderung des Staph. aureus-Befundes vor der Triclosan/Flumetasonpivalat-Behandlung im Vergleich zum Ende der Therapie wurde als Nebenzielkriterium festgelegt.

Vor Therapiebeginn sowie zum Behandlungsende wurde zudem die Lebensqualität der Patienten mit dem Dermatology Life Quality Index (DLQI) [25] erhoben. Der DLQI ist ein Bewertungsinstrument zur Beurteilung der Lebensqualität (bzw. deren Beeinträchtigung) bei Hauterkrankungen durch den Patienten selbst. Der Index umfasst 10 Fragen zum Ausmaß der Beeinträchtigung in sechs verschiedenen Lebensbereichen (Symptome, tägliche Aktivitäten, Freizeit/Sport, Arbeit/Schule, Beziehungen, Therapie) in den vorhergegangenen 7 Tagen. Insgesamt können Werte zwischen 0 und 30 (0-1 [keine Beeinträchtigung], 2 - 5 [geringe Beeinträchtigung], 6-10 [mittelstarke Beeinträchtigung], 11-20 [sehr große Beeinträchtigung], 21 - 30 [extrem große Beeinträchtigung]) erreicht werden.

Daneben wurde mit dem Patient Benefit Index (PBI) [26] der patientendefinierte Nutzen erfasst. Der PBI ist ein Fragenbogen zur Erfassung patientenseitiger Therapieziele und -nutzen im Verlauf einer Therapie. Die Patienten werden vor der Therapie mit einem strukturierten Fragebogen gebeten, die Wichtigkeit von 23 vorgegebenen Therapiezielen anhand einer fünfstufigen Skala ( 0 = gar nicht wichtig, $1=$ etwas wichtig, $2=$ mäßig wichtig, $3=$ ziemlich wichtig, $4=$ sehr wichtig) einzuschätzen. Nach Abschluss der Therapie beurteilen die Patienten das Ausmaß der Erreichung dieser patientenseitigen Therapieziele $(0=$ gar nicht erreicht, 1 =etwas erreicht, 2 =mäßig erreicht, $3=$ ziemlich erreicht, $4=$ sehr erreicht). Für jeden Patienten wird ein einzelner Indexwert ( $0=$ kein Nutzen, $4=$ maximal möglicher Nutzen) errechnet, bei dem das Ausmaß des Erreichens der Therapieziele anhand deren individueller Wichtigkeit zu Beginn der Therapie gewichtet wird. Ein höherer Indexwert resultiert somit, wenn diejenigen Beschwerden gelindert werden, deren Verbesserung für den Patienten zu Behandlungsbeginn besonders wichtig war. Die Patienten schätzten außerdem nach 7 Tagen sowie zum Therapieende die Wirksamkeit und die Verträglichkeit der Behandlung jeweils auf einer fünfstufigen Skala $(-2=$ sehr schlecht, - 1 = schlecht, 0 = mäßig, 1 = gut, 2 = sehr gut) ein.

Die statistische Auswertung erfolgte mit SPSS, Version 15. Die Verbesserung der Symptomschwere (vor Therapie vs. Therapieende) wurde mit dem t-Test für abhängige Stichproben auf Signifikanz getestet. Die Veränderung der Häufigkeit einer Staph. aureus-Besiedlung im Prüfareal vor Therapie im Vergleich zum Therapieende wurde mit dem $X^{2}$-Test von McNemar überprüft. Die Irrtumswahrscheinlichkeit wurde nach Alpha-Adjustierung nach Bonferroni [27] auf $p<0,025$ gesetzt. Alle übrigen Zielkriterien wurden deskriptiv ausgewertet. 


\section{Ergebnisse}

$\nabla$

Von $\mathrm{n}=29$ der 31 Patienten lagen zum Zeitpunkt der Auswertung alle ausgefüllten Fragebogen von Arzt und Patient über den zwei-

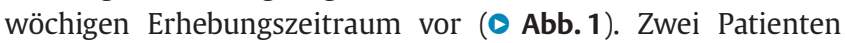
schieden vorzeitig aus nicht-medizinischen Gründen aus der Studie aus. Es wurden gemäß Intention-to-treat (ITT)-Analyse alle 31 Patienten in die Auswertung einbezogen.

\section{Soziodemografische und krankheitsbezogene Daten}

Soziodemografische und krankheitsbezogene Daten der Patienten sind in $\bullet$ Tab. 1 angeführt. Das durchschnittliche Alter der eingeschlossenen Patienten betrug 30 Jahre, 15 Patienten (48\%) waren männlich, 16 Patienten weiblich (52\%). Die mittlere Krankheitsdauer lag bei 15 Jahren. 5 Patienten (16\%) wiesen Begleiterkrankungen auf ( Tab. 2).

\section{Klinischer Befund: Schweregrad der klinischen Symptome}

Vor Behandlungsbeginn wiesen die Patienten im Prüfareal einen mittleren Symptomscore von 1,14 \pm 0,39 auf, der nach 14 Tagen topischer Therapie mit der Triclosan/Flumetasonpivalat-Kombination signifikant auf 0,43 $\pm 0,29$ absank ( $p<0,001$; Abb. 2 a).

- Abb. 2 b zeigt die Ausprägung der sechs in den klinischen Score einfließenden Symptome vor Therapiebeginn und im Verlauf der Behandlung. Bereits nach einer Woche (Tag 7) zeigte sich für alle Einzelsymptome eine Reduktion der Symptomschwere.

\section{Mikrobiologischer Befund}

Eine Besiedlung mit Staph. aureus im Prüfareal lag vor Therapiebeginn bei 17 (55\%) Patienten vor. Staph. aureus war damit der am häufigsten nachweisbare Keim, gefolgt von Staph. epidermidis, der bei 11 Patienten (36\%) nachgewiesen wurde. Der Anteil der Patienten, bei denen im Prüfareal Staph. aureus nachgewiesen wurde, nahm im Verlauf der zweiwöchigen Behandlung signifikant ab $(p<0,01)$. So war der Keim nach einer Woche Therapie (Tag 7) nur noch bei 9 Patienten (30\%) und zum Behandlungsende (Tag 14) bei 8 Patienten (28\%) nachweisbar.

Die Schwere der klinischen Symptome im Prüfareal verringerte sich bei Patienten mit Nachweis von Staph. aureus in ähnlichem Maße (vor Therapie: $1,14 \pm 0,41$; Therapieende: $0,49 \pm 0,34$ ) wie bei den Patienten, bei denen Staph. aureus nicht nachgewiesen wurde (vor Therapie: 1,14 $\pm 0,39$; Therapieende: 0,35 $\pm 0,21$ ) Der Anteil der Patienten mit Staph. epidermis-Besiedlung im Prüfareal nahm im Verlauf der Behandlung nicht signifikant ab.

\section{Patientenbewertung der klinischen Wirksamkeit}

Die Wirksamkeit der Behandlung $(-2=$ sehr schlecht, $-1=$ schlecht, 0 = mäßig, 1 = gut, 2 = sehr gut) wurde von den Patienten nach 7 Tagen und zum Abschluss der zweiwöchigen Therapie im Mittel als „gut“ eingestuft (Tag 7: 1,07 $\pm 0,64$; Therapieende: $1,11 \pm 0,89$ ).

\section{Patientendefinierter Nutzen}

Die drei wichtigsten Therapieziele des mit dem Patient Benefit Index (PBI) gemessenen patientendefinierten Nutzens waren für die Patienten „Keinen Juckreiz mehr zu empfinden“, „Von allen Hautveränderungen geheilt zu sein“ und „Vertrauen in die Therapie zu haben“. In $\bullet$ Tab. 3 sind die einzelnen Therapieziele vor Behandlungsbeginn gemäß ihrer Wichtigkeit für die Patienten sowie das Erreichen dieser Ziele durch die zweiwöchige Behandlung dargestellt. Der patientendefinierte Therapienutzen (PBI:

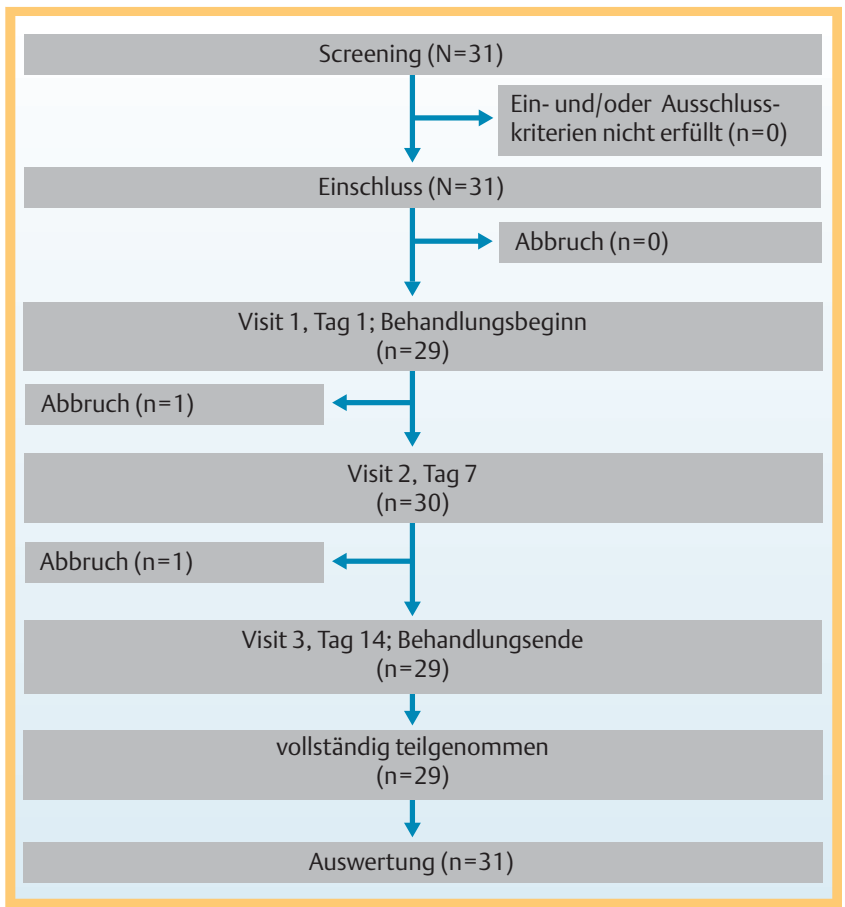

Abb.1 Im Flussdiagramm sind die Anzahl der Patienten für die drei Messzeitpunkte (vor Therapie, Tag 7 und Tag 14) dargestellt.

Tab. 1 Soziodemografische und anamnestische Daten.

\begin{tabular}{|l|l|}
\hline Geschlecht & $\begin{array}{l}\text { Häufigkeit (Prozent) } \\
\text { männlich: 15 (48\%) } \\
\text { weiblich: } 16(52 \%)\end{array}$ \\
\hline $\begin{array}{l}\text { Mittelwert } \pm \text { SD } \\
\text { Alter }\end{array}$ & $30,1 \pm 16,4$ \\
\hline Global Assessment (Arzturteil): & \\
Schweregrad der Akne zu T1 & 3,8 \\
$\quad$ gering & 30,5 \\
mäßig & 60,5 \\
mittel & 5,1 \\
schwer & 0,1 \\
sehr schwer & 2,2 \\
\hline Missing & $15,0 \pm 16,0$ \\
\hline Alter bei Erstauftreten & $15,1 \pm 12,8$ \\
\hline Krankheitsdauer in Jahren & $4,3 \pm 2,8$ \\
\hline Anzahl Schübe letzte 5 Jahre & \\
\hline
\end{tabular}

Tab. 2 Begleiterkrankungen.

\begin{tabular}{lll} 
Begleiterkrankungen & Häufigkeit & (Prozent) \\
\hline Patienten mit Begleiterkrankungen & 5 & $(16 \%)$ \\
\hline Art der Begleiterkrankung & Häufigkeit & (Prozent) \\
\hline Asthma bronchiale & 3 & $(10 \%)$ \\
Heuschnupfen & 1 & $(3 \%)$ \\
\hline Hypertonie & 1 & $(3 \%)$ \\
\hline Rhinokonjuntivitis allergica & 1 & $(3 \%)$ \\
\hline Plantarwarze & 1 & $(3 \%)$
\end{tabular}

$0=$ kein Nutzen, $4=$ maximal möglicher Nutzen) betrug durchschnittlich 3,21. Somit konnten die für die Patienten wichtigen Therapieziele unter der zweiwöchigen Therapie mit Triclosan/ Flumetasonpivalat weitgehend erreicht werden. 


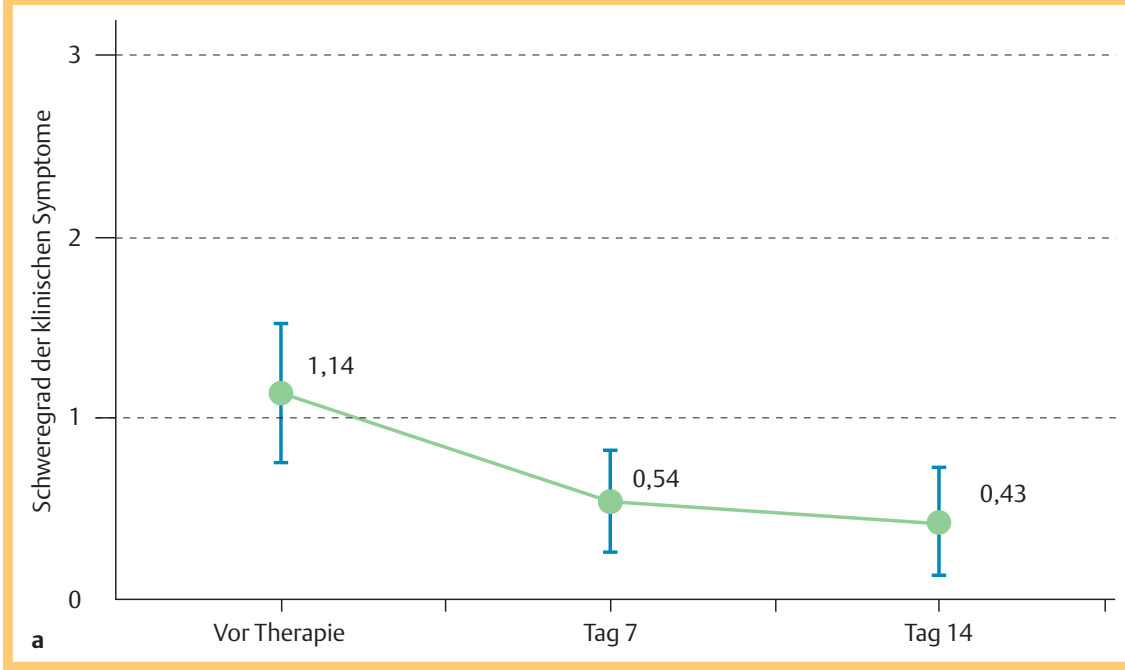

Abb. 2 a Verlauf des mittleren Schweregrades des klinischen Symptomscores im Prüfareal während der zweiwöchigen Behandlung. $0=$ keine Symptome, 1 = leichte Symptome, 2 = mäßige Symptome, 3 = starke Symptome vor Therapie vs. Behandlungsende. Der mittlere Schweregrad nahm signifikant ab $(p<0,001)$.

b Verlauf des mittleren Schweregrades der 6 klinischen Symptome im Prüfareal während der zweiwöchigen Behandlung. 0 = keine Symptome, 1 = leichte Symptome, 2 = mäßige Symptome, 3 = starke Symptome.

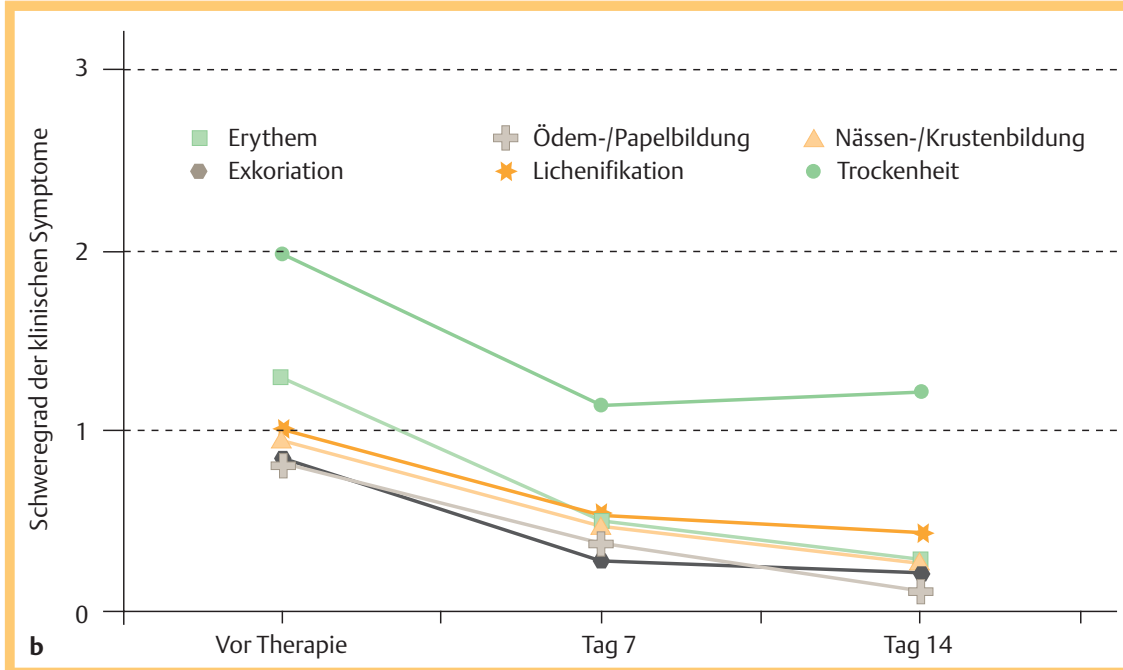

\section{Lebensqualität}

Die mit dem DLQI gemessene Lebensqualität nahm im Laufe der Therapie zu. Vor Therapiebeginn lag der durchschnittliche DLQI bei 8,13 $\pm 4,78$ und fiel nach der zweiwöchigen Behandlung der atopischen Dermatitis im Mittel auf 3,21 $\pm 3,59$. Der Verlauf des DLQI unter der Therapie ist in $\bullet$ Abb. $\mathbf{3}$ dargestellt.

\section{Sicherheit, Verträglichkeit}

Es traten keine unerwünschten Ereignisse (AEs) auf. Die Verträglichkeit der Behandlung $(-2=$ sehr schlecht, $-1=$ schlecht, $0=$ mäßig, 1 = gut, $2=$ sehr gut) wurde von den Patienten im Mittel als „gut" bis „sehr gut“ bewertet (nach 7 Tagen: 1,57 $\pm 1,52$; Behandlungsende: 1,52 $\pm 0,64$ ).

\section{Diskussion}

Leichte bis mittelstark wirksame Kortikosteroide sind die wichtigste antientzündliche Lokaltherapie der atopischen Dermatitis [14]. Der topische Einsatz der Calcineurininhibitoren Pimecrolimus und Tacrolimus wird dagegen aufgrund ihres Nebenwirkungs- [28] und Kosten-Nutzen-Profils teilweise kritisch bewertet $[29,30]$. Vor dem Hintergrund der Bedeutung einer bakteriellen Superinfektion als pathophysiologisch relevanten und krankheitsunterhaltenden Faktor des atopischen Ekzems [1] haben sich antimikrobielle Substanzen als zusätzliches Therapieprinzip etabliert $[6,31,32]$. In der Mehrzahl der Untersuchungen waren Kombinationspräparate aus Kortikosteroid und antimikrobiell wirksamer Substanz in der topischen Anwendung einer Monotherapie mit Kortikosteroiden überlegen [33-35]. Allerdings muss beim Einsatz lokaler Antibiotika gerade bei Staph. aureus mit der Entwicklung von Resistenzen gerechnet werden [35]. Hier bietet sich die Anwendung eines Antiseptikums wie Triclosan an [16]. Im Direktvergleich mit beispielsweise Erythromycin zeigte sich Triclosan als wirkungsäquivalent [15]. Insbesondere eine Besiedlung mit Staph. aureus konnte vollständig beseitigt werden.

In der vorliegenden Anwendungsbeobachtung erwies sich die Kombination von Triclosan mit Flumetasonpivalat als gut wirksam in der Therapie des atopischen Ekzems. Parallel zur Reduktion der klinischen Symptome fand sich im Prüfareal eine signifikante Abnahme der Besiedlung mit Staph. aureus, während die Häufigkeit der Besiedlung mit Staph. epidermidis nicht abnahm. Allerdings war die Reduktion der klinischen Symptome vergleichbar bei Patienten mit und ohne Nachweis von Staph. aureus. Zur weiteren Klärung der Bedeutung des Zusatzes von Triclosan wären daher Untersuchungen interessant, die eine Flumetasonpivalat-Monotherapie mit der Triclosan/FlumetasonpivalatKombinationstherapie bei Patienten mit einer Staph. aureus Besiedlung vergleichen. Dabei sollte in einer solchen Studie auch 
Tab.3 Wichtigkeit der Therapieziele vor Behandlungsbeginn und Erreichen der Ziele zum Behandlungsende.

\begin{tabular}{|c|c|c|}
\hline Therapieziele & $\begin{array}{l}\text { Wichtigkeit } \\
\text { (vor Therapie) } \\
\text { M } \pm \text { SD }\end{array}$ & $\begin{array}{l}\text { Erreicht } \\
\text { (nach Therapie) } \\
M \pm S D\end{array}$ \\
\hline 1. Keinen Juckreiz mehr zu empfinden & $3,72 \pm 0,68$ & $2,96 \pm 1,17$ \\
\hline 2. Von allen Hautveränderungen geheilt zu sein & $3,67 \pm 0,56$ & $2,08 \pm 1,38$ \\
\hline 3. Vertrauen in die Therapie zu haben & $3,44 \pm 1,04$ & $2,52 \pm 2,57$ \\
\hline 4. Kein Brennen an der Haut mehr zu haben & $3,43 \pm 0,98$ & $3,00 \pm 1,00$ \\
\hline 5. Eine klare Diagnose und Therapie zu finden & $3,40 \pm 0,96$ & $2,64 \pm 1,50$ \\
\hline 6. Keine Furcht vor einem Fortschreiten der Krankheit & $3,30 \pm 1,06$ & $2,65 \pm 1,40$ \\
\hline 7. Weniger eigene Behandlungskosten zu haben & $3,29 \pm 1,12$ & $2,79 \pm 1,27$ \\
\hline 8. Mehr Kontakte mit anderen Menschen haben zu können & $3,19 \pm 1,11$ & $2,75 \pm 1,24$ \\
\hline 9. Sich mehr zeigen zu mögen & $3,14 \pm 1,15$ & $2,26 \pm 1,29$ \\
\hline 10. Ein normales Alltagsleben führen zu & $3,14 \pm 1,13$ & $2,85 \pm 1,35$ \\
\hline 11. Weniger Zeitaufwand mit der tägl. Behandlung zu haben & $3,09 \pm 1,08$ & $2,76 \pm 1,41$ \\
\hline 12. Schmerzfrei zu sein & $3,05 \pm 1,23$ & $2,80 \pm 1,20$ \\
\hline 13. Weniger Nebenwirkungen zu haben & $3,04 \pm 1,19$ & $2,67 \pm 1,43$ \\
\hline 14. Im Alltag leistungsfähiger zu sein & $3,00 \pm 1,20$ & $2,56 \pm 1,34$ \\
\hline 15. Besser schlafen zu können & $2,96 \pm 1,22$ & $2,20 \pm 1,51$ \\
\hline 16. Weniger auf Arzt- und Klinikbesuche angewiesen zu sein & $2,87 \pm 1,32$ & $2,17 \pm 1,47$ \\
\hline 17. An Lebensfreude zu gewinnen & $2,71 \pm 1,21$ & $2,50 \pm 1,26$ \\
\hline 18. Normalen Freizeitaktivitäten nachgehen zu können & $2,60 \pm 1,39$ & $2,79 \pm 1,32$ \\
\hline 19. Ein normales Berufsleben führen zu können & $2,44 \pm 1,50$ & $2,24 \pm 1,52$ \\
\hline 20. Weniger niedergeschlagen zu sein & $2,42 \pm 1,57$ & $2,44 \pm 1,21$ \\
\hline 21. In der Partnerschaft weniger belastet zu sein & $2,41 \pm 1,37$ & $2,44 \pm 1,03$ \\
\hline 22. Ein normales Sexualleben führen zu können & $2,33 \pm 1,54$ & $2,31 \pm 1,32$ \\
\hline 23. Meine Angehörigen und Freunde weniger zu belasten & $2,00 \pm 1,67$ & $2,93 \pm 1,14$ \\
\hline
\end{tabular}

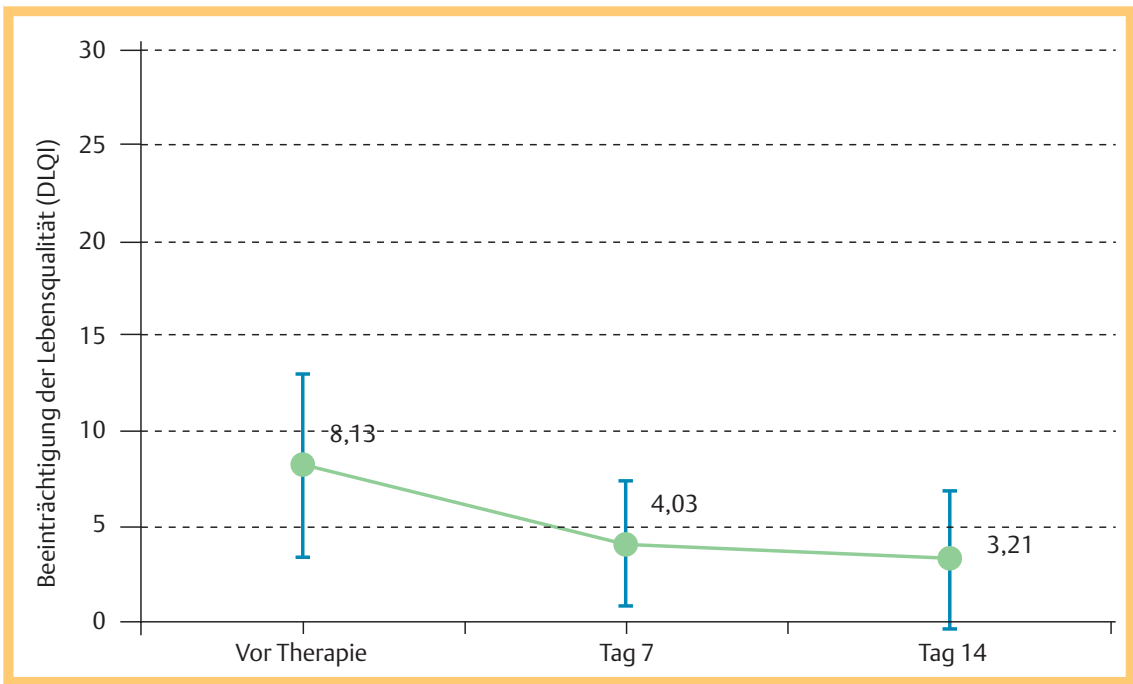

Abb. 3 Verlauf des mittleren DLQI-Gesamtscores während der zweiwöchigen Behandlung. $0-1=$ keine Beeinträchtigung, $2-5=$ geringe Beeinträchtigung, $6-10=$ mittelstarke Beeinträchtigung, $11-20=$ sehr große Beeinträchtigung, $21-30=$ extrem große Beeinträchtigung.

die Zeit bis zum Rezidiv nach Therapieende zwischen beiden Therapien in Abhängigkeit von der Eradikation des Keims untersucht werden.

Limitationen dieser Anwendungsbeobachtung liegen in der relativ kleinen Fallzahl und einer fehlenden Kontrollgruppe. Daneben wurde die Therapiewirkung in einem definierten Prüfareal und nicht anhand eines validierten Scores wie z.B. dem SCORAD untersucht, der eine bessere Vergleichbarkeit mit anderen Studiendaten gewährleistet hätte. Der Vorteil dieser Anwendungsbeobachtung liegt in einer Therapie unter realen klinischen Bedingungen einer Hautarztpraxis mit nur sehr geringer Selektion der Patienten.

\section{Fazit für die Praxis}

Diese Anwendungsbeobachtung hat Hinweise dafür erbracht, dass die fixe Kombination aus Triclosan und Flumetasonpivalat zur klinischen Besserung der atopischen Dermatitis und Reduktion der Besiedlung mit Staph. aureus bei günstigem Nutzen-Risiko-Profil eingesetzt werden kann.

\section{Interessenkonflikt}

Die Durchführung dieser Studie wurde von der GALENpharma GmbH gesponsert. Der Sponsor hatte keinen Einfluss auf die Durchführung, Auswertung oder Interpretation der Studie. 


\section{Efficacy of a Combined Treatment of Atopic Dermatitis with Triclosan and Flumethasone Pivalate (DuoGalen ${ }^{\circledR}$ Cream)}

Objective: The goal of this observational study was to evaluate the efficacy and patient-reported benefit of a fixed combination of triclosan and flumethasone pivalate in the treatment of atopic dermatitis.

Patients and Method: 31 patients with mild to moderate atopic dermatitis were enrolled in the observational study, in which triclosan/flumethasone pivalate was applied twice daily for two weeks. The severity of clinical symptoms and bacterial colonisation of a test area were evaluated. Patients assessed the effectiveness, tolerability and subjective therapeutic benefit of the treatment as well as the disease-related quality of life.

Results: The clinical symptom score $(0=$ no symptoms, $1=$ mild, 2 = moderate, 3 = severe symptoms) decreased significantly in the course of the triclosan/flumethasone pivalate treatment (before therapy: $1.14 \pm 0.39$; end of therapy: $0.43 \pm 0.29 ; p<0.001)$. In parallel, there was a reduction of the test area infected with Staphylococcus aureus (decrease from $55 \%$ to $28 \%$; $p<0.01$ ). The efficacy of the therapy was rated on average by the patients as „good“, the tolerability as „good“ to „very good“. The quality of life improved considerably (DLQI; before therapy: $8.13 \pm 4.78$; end of therapy: $3.21 \pm 3.59$ ).

Conclusions: The results are consistent with a good clinical efficacy and antiseptic activity of triclosan/flumethasone pivalate in the treatment of atopic dermatitis combined with high tolerability and patient satisfaction.

\section{Literatur}

1 Hanifin JM, Rajka G. Diagnostic features of atopic dermatitis. Acta Derm Venereol (Stockh) 1980; 92: 44-47

2 Schäfer T, Borowski C, Diepgen TL und weitere Mitlieder der Konsensusgruppe des Aktionsbündnisses Allergieprävention. Evidenzbasierte und konsentierte Leitlinie des Aktionsbündnisses Allergieprävention. Allergo J 2004; 13: $252-260$

3 Kiebert G, Sorensen SV, Revicki D, Fagan SC, Doyle JJ, Cohen J, Fivenson D. Atopic dermatitis is associated with a decrement in health-related quality of life. Int J Dermatol 2002; 41: 151 -158

4 Holm EA, Wulf HC, Stegmann H, Jemec GB. Life quality assessment among patients with atopic eczema. Br J Dermatol 2006; 154: 719725

5 Leung DY, Boguniewicz M, Howell MD, Nomura I, Hamid QA. New insights into atopic dermatitis. J Clin Invest 2004; 113: 651-657

6 Abeck D, Mempel M. Staphylococcus aureus colonization in atopic dermatitis and its therapeutic implications. Br J Dermatol 1998; 139: 13 16

7 Aly R. Bacteriology of atopic dermatitis. Acta Derm Venereol 1980; 92: $16-18$

8 Hauser C, Wuethrich B, Matter L, Wilhelm JA, Sonnabend W, Schopfer K. Staphylococcus aureus skin colonization in atopic dermatitis. Dermatology 1985; 170: 35- 39

9 Leung DYM, Harbeck R, Bina P, Reiser R, Yang E, Norris DA. Presence of IgE antibodies to staphylococcal exotoxins on the skin of patients with atopic dermatitis. J Clin Invest 1993; 92: 1374-1380

10 Leyden JE, Marples RR, Kligmann AM. Staphylococcus aureus in the lesions of atopic dermatitis. Br J Dermatol 1974; 90: 525-530

11 Breuer K, Häussler S, Kapp A, Werfel T. Staphylococcus aureus: colonizing features and influence of an antibacterial treatment in adults with atopic dermatitis. Br J Dermatol 2002; 147: 55-61
12 Williams REA, Gibson AG, Aitchison TC, Lever R, Mackie RM. Assessment of contact-plate sampling technique and subsequent quantitative bacterial studies in atopic dermatitis. Br J Dermatol 1990; 123: 493 - 501

13 Bunikowski R, Mielke M, Skarabis H, Worm M, Anagnostopoulos I, Kolde $G$, Wahn U, Renz H. Evidence for a diseasepromoting effect of Staphylococcus aureus-derived exotoxins in atopic dermatitis. J Allergy Clin Immunol 2000; 105: 814-819

14 Akdis CA, Akdis M, Bieber T et al. Diagnosis and treatment of atopic dermatitis in children and adults: European Academy of Allergology and Clinical Immunology/American Academy of Allergy, Asthma and Immunology/PRACTALL Consensus Report. J Allergy Clin Immunol 2006; 118: $152-169$

15 Gehring W, Forssman T, Jost G, Gloor M. Die keimreduzierende Wirkung von Erythromycin und Triclosan bei der atopischen Dermatitis. Akt Dermatol 1996; 22: 28-31

16 Gloor M, Becker A, Wasik B, Kniehl E. Triclosan, ein dermatologisches Lokaltherapeutikum. Hautarzt 2002; 53: $724-729$

17 Bhargava HN, Leonard PA. Triclosan: Applications and safety. Am J Infect Control 1996; 24: 209-218

18 Allegra F, Cainelli T, Csermely E et al. Statistically evaluated clinical observations from a multicentre study on the activity of a new topical antimicrobial and antimycotic agent in paediatric skin diseases. Panminerva Med 1978; 20: 239-249

19 Reiffers J. Clinical report of triclosan, a new topical with antifungicidal and antimicrobial activity. Schweiz Rundsch Med Prax 1981; 70 : $1050-1053$

20 Roubicek M, Krebs A. Logamel, ein neues Lokaltherapeutikum bei infizierten entzündlichen Dermatosen. Ergebnisse einer kontrollierten Prüfung bei akuten Dermatomykosen. Schweiz Rundsch Med Prax 1977; 66: $585-588$

21 Sampaio SA, Jardim ML, Bartelle CJ et al. Treatment of infected eczematous dermatitis with an association of triclosan and flumethasone. Folha méd 1981; 82: 295-297

22 Weitgasser H. Erfahrungen mit magistraliter zubereiteten Verdünnungen eines neuen Flumethasonpivalat-Triclosan-Dermatikums in der dermatologischen Praxis: Ergebnisse einer multizentrischen klinischen Prüfung. Wien Med Wochenschr 1979; 129: 193-197

23 Victor $N$, Windeler J, Hasford J et al. Recommendations concerning the conduct of „Anwendungsbeobachtungen“. Informatik, Biometrie und Epidemiologie in Medizin und Biologie 1997; 4: 247-252

24 European Task Force on Atopic Dermatitis: Severity scoring of atopic dermatitis: the SCORAD index. Consensus Report of the European Task Force on Atopic Dermatitis. Dermatology 1993; 186: 23 - 31

25 Finlay AY, Khan GK. Dermatology Life Quality Index (DLQI) - A simple practical measure for routine clinical use. Clinical and Experimental Dermatology 1994; 19: 210-216

26 Augustin M, Reich C, Schaefer I, Zschocke I, Rustenbach SJ. Development and validation of a new instrument for the assessment of patientdefined benefit in the treatment of acne. J Dtsch Dermatol Ges 2008; 6: $113-120$

27 Bortz J. Statistik. 4. Auflage. Berlin: Springer, 1993

28 Furue M, Terao H, Rikihisa W, Urabe K, Kinukawa N, Nose Y, Koga T. Clinical dose and adverse effects of topical steroids in daily management of atopic dermatitis. Br J Dermatol 2003; 148: 128-133

29 Buys LM. Treatment options for atopic dermatitis. Am Fam Physician 2007; 75: $523-528$

30 Bigby M. Tacrolimus and pimecrolimus for atopic dermatitis: where do they fit in. Arch Dermatol 2006; 142: $1203-1205$

31 Wachs GN, Maibach HI. Co-operative double-blind trial of an antibiotic/ corticoid combination in impetiginized atopic dermatitis. Br J Dermatol 1976; 95: $323-328$

32 Hjorth N, Schmidt H, Thomsen K. Fusidic Acid plus betamethasone in infected or potentially infected eczema. Pharmatherapeutica 1985; 4: $126-131$

33 Javier PR, Ortiz M, Torralba $M$ et al. Fusidic acid/betamethasone in infected dermatoses-a double-blind comparison with neomycin/betamethasone. Br J Clin Pract 1986; 40: 235-238

34 Larsen FS, Simonsen L, Melgaard A, Wendicke K, Henriksen AS. An efficient new formulation of fusidic acid and betamethasone 17-valerate (fucicort lipid cream) for treatment of clinically infected atopic dermatitis. Acta Derm Venereol 2007; 87: $62-68$

35 Stalder JF, Fleury $M$, Sourisse $M$ et al. Local steroid therapy and bacterial skin flora in atopic dermatitis. Br J Dermatol 1994; 131: 536-540 